\title{
Using the ICF to clarify team roles and demonstrate clinical reasoning in
}

stroke rehabilitation.

\section{Authors and Affiliations:}

Stephanie Tempest MSc BSc (Hons)

Lecturer in Occupational Therapy

Brunel University

Anne McIntyre MSc, PGCertTLHE, DipCOT

Lecturer in Occupational Therapy

Brunel University 


\section{Clinical Commentary}

\section{Using the ICF to clarify team roles and demonstrate clinical reasoning in stroke rehabilitation.}

\section{$\underline{\text { Full reference }}$}

Tempest S, Mclntyre A (2006) Using the ICF to clarify team roles and demonstrate clinical reasoning in stroke rehabilitation. Disability and Rehabilitation 28 (10) pp 663 -667 


\section{Abstract}

Purpose: The International Classification of Functioning, Disability and Health (ICF) is advocated as a tool to structure rehabilitation and a universal language to aid communication, within the multi-disciplinary team (MDT). The ICF may also facilitate clarification of team roles and clinical reasoning for intervention. This article aims to explore both factors in stroke rehabilitation.

Method: Following a review of the literature, a summary was presented and discussed with clinicians working within stroke rehabilitation, to gather expert opinions. The discussions were informal, being part of service development and on-going education. The clinicians summarised key themes for the potential use of the ICF within clinical practice.

Results: Two key themes emerged from the literature and expert opinion for the potential use of the ICF in stroke rehabilitation: i) to aid communication and structure service provision ii) to clarify team roles and aid clinical reasoning. Expert opinion was that clarification of team roles needs to occur at a local level due to the skill mix, particular interests, setting and staffing levels within individual teams. The ICF has the potential to demonstrate/ facilitate clinical reasoning, especially when different MDT members are working on the same intervention. 
Conclusion: There is potential for the ICF to be used to clarify team roles and demonstrate clinical reasoning within stroke rehabilitation. Further experiential research is required to substantiate this view.

\section{Introduction}

Evidence has demonstrated that stroke patients who receive organised in-patient care are more likely to survive, return home and make a good recovery ${ }^{1}$. There is continued debate regarding what constitutes well-organised care and it has not been established why stroke units are effective ${ }^{2}$. There are many factors that may promote effectiveness within stroke services and the Intercollegiate Stroke Working Party ${ }^{3}$ has developed recommendations for good practice based on the available evidence. The recommendations include a structured service with regular team meetings, establishing a common language to aid communication using the terminology within the International Classification of Functioning, Disability and Health (ICF) ${ }^{4}$ and team members who understand their own roles within the multidisciplinary team (MDT).

There is increasingly stronger emphasis for evidence-based practice and, as a result, therapists have to justify more robustly their place in the healthcare team 5. At a clinical level, it is essential to have a clear understanding of professional boundaries and a mutual respect for the skills and abilities of other health care professionals, to enhance professional working relationships ${ }^{6}$. More importantly, professional groups involved in stroke care need to reconsider their role within 
the MDT and how they can work together to improve outcomes for patients and their families ${ }^{3}$.

It has been recommended that the ICF is a useful framework to aid communication and also as a structure to plan rehabilitation programmes ${ }^{7}$. However, there is further scope for the ICF framework and classification and the aim of this article is to explore the literature and expert opinion, regarding the use of the ICF to clarify team roles and demonstrate clinical reasoning.

This article does not attempt to describe the ICF and readers are encouraged to use other resources including the World Health Organisation (WHO) website (http://www3.who.int/icf/icftemplate.cfm) and previous work in this journal ${ }^{8,9}$

\section{Method}

A literature search was conducted using the following headings: ICF, theoretical models, World Health Organisation, rehabilitation, stroke, cardiovascular accident, physical therapy, occupational therapy, nursing, roles, role overlap, clinical reasoning and intervention. Ebscohost EJS, Medline, Cinahl and Ovid databases were used and further searches included the ICF homepage on the WHO website and a review of reference lists from the pertinent articles.

A number of presentations were given to clinicians working within stroke rehabilitation regarding the ICF framework and classification, by both authors, 
incorporating the findings from the review of the literature. The information gathering was informal, as part of working in clinical practice, facilitating inservice teaching, post-graduate teaching on the ICF and whilst implementing the framework within a local occupational therapy service and stroke service. Discussions followed each presentation with expert opinions gathered from nursing staff, occupational therapists, physiotherapists, speech and language therapists and dieticians. The clinicians were asked to summarise the key themes from the presentations and discussions and these formed the basis of the expert opinions.

Opinion was gathered from colleagues as part of practice evaluation and service development. Formal ethical approval was therefore not required at the time of collecting data. However as a result of the intention to publish, permission was subsequently obtained from the services involved.

\section{Results}

Two key themes emerged from the literature and the expert opinions regarding the potential use of the ICF within stroke rehabilitation, firstly, to aid communication and structure service provision and secondly to clarify team roles and aid demonstration of clinical reasoning, especially when there appears to be role overlap. 
Aiding communication and structure service provision

There is considerable evidence in the literature to support the use of the ICF to structure stroke services and to adopt the framework to aid communication within the MDT,3,7,9,10,11,12,15. Expert opinion supported this view although acknowledged that it could be a lengthy process as, to begin with, clinicians needed to be made aware of and understand the ICF framework and the core set for stroke before considering its application.

Clarify team roles and demonstrate clinical reasoning

Evidence exists in the literature to support the use of the ICF to clarify team roles 12,13,17 and emerging literature supports the use of the ICF to facilitate clinical reasoning ${ }^{10}$. The general view from the clinicians supported use of the ICF to clarify team roles, as it is felt in practice that there continues to be a lack of understanding regarding "who does what", which negatively impacts on the quality of service provision. It was also acknowledged that whilst on face value, duplication of intervention was seemingly carried out by different MDT members, the clinical reasoning differed and therefore also the goals of intervention. The ICF could help to communicate such differences and aid greater understanding of the necessity of role overlap within stroke rehabilitation.

\section{Discussion}

The scope of the ICF extends beyond a useful tool to aid effective communication as it is also designed as a tool for clinical practice ${ }^{14}$. A particular 
advantage of the ICF is that it transcends uni-disciplinary frameworks and models, offering a bio-psycho-social model for health and social care. A recognised disadvantage is that the complete classification system involves a lengthy process to use within practice and this might discourage the uptake of the ICF. Therefore, core sets have been developed including a specific core set for stroke ${ }^{15}$ and stage specific core sets e.g. for patients with neurological conditions in early post-acute rehabilitation facilities ${ }^{18}$. It is conceivable that the relevant core sets could be used to identify team roles within stroke rehabilitation. There is considerable evidence to support the use of the ICF to aid communication and structure service provision $3,7,9,10,11,12,15$. The evidence for the use of the ICF to clarify team roles and aid clinical reasoning, is at an emergent stage and therefore these themes will be the focus for this discussion.

\section{The ICF to clarify team roles}

The need to examine the role overlap and core business for different professions in stroke rehabilitation does not stem from a need to be overprotective of individual roles. It is recognised that role overlap is a complex issue and the way it is managed affects the extent and success of interprofessional working in stroke rehabilitation ${ }^{16}$.

Rentsch et $\mathrm{al}^{12}$ used the activity domains within the ICF to identify which member of the MDT took the lead with particular aspects of the rehabilitation process. For example, the occupational therapist took responsibility for reporting on four 
domains: Learning and Applying knowledge, Domestic Life, General Tasks and demands and Mobility (jointly with the physiotherapist). Therefore, each member of the team took lead responsibility for different aspects within the rehabilitation process, ensuring the needs of the stroke survivor were met. Leading on from this, they structured feedback in rehabilitation meetings around the activity domains and the member of the MDT who led on the particular domain also led the feedback within meetings. The team concluded that using the ICF considerably improved the quality of work and the interdisciplinary work process. It contributed a systematic approach and a basis for effective MDT communication ${ }^{12}$.

Steiner et $\mathrm{al}^{13}$ discussed how an MDT used the ICF model to develop a Rehabilitation Problem-Solving Form. This incorporated ICF terminology and was used by the MDT with the patient, to identify the specific target problems and plan appropriate intervention. The team acknowledged that the ICF provided a structure to facilitate intra and interprofessional communication and considered the model to be the future tool for organising information about functioning and disabilities ${ }^{13}$.

However at a uni-disciplinary level, ICF categories have also been identified for nursing interventions with neurological patients ${ }^{17}$ thus highlighting the potential to clarify individual roles within the MDT stroke rehabilitation team. 
These examples demonstrate that using the ICF to structure rehabilitation services consequentially provides clarification for each profession to identify their own role within the process.

Informal discussion with colleagues has highlighted that the role overlap between professions varies according to the work setting (e.g. acute care or the community), as well as the interests and skills of individual therapists and staffing levels within the team. Therefore, the ICF could be used at a local level by specific stroke services to identify which member of the team will take the lead on each domain. This could enable effective service delivery by clarifying the individual roles within the team and avoid duplication of interventions.

The advantages for multi-disciplinary working identified by Rentsch et $\mathrm{al}^{12}$ and Steiner et $\mathrm{al}^{13}$ also extends to inter-agency working and service development. It has been acknowledged that there is potential for the ICF to clarify roles and responsibilities across services ${ }^{10}$. The ICF is recommended as a framework to organise service delivery across health and social care and this is supported by theoretical and practical examples of application ${ }^{10}$.

Within stroke services, the framework and classification has the potential not only to identify "who does what" within each team, but also which services cannot be provided. For example, Major Life Areas (Chapter 8 within Activities and Participation $)^{4}$ includes seeking employment, vocational training and full time 
employment. These areas may not be possible to address within an early postacute stroke rehabilitation setting. However if there are inadequate community resources to address the particular need of a stroke survivor, this is an unmet need within the system. By using the ICF structure, stroke services can acknowledge gaps in their service provision, thus adding to the evidence base for business cases for future developments.

\section{Using the ICF to demonstrate clinical reasoning}

Expert opinion also stipulated that the ICF could also be used to demonstrate the different clinical reasoning for members of the team working in collaboration on the same activity e.g. maintaining sitting position in acute stroke management. The physiotherapist may address maintaining a sitting position (activity) to maintain muscle tone functions (body function). A speech and language therapist may jointly review the same activity to assess swallowing function (body function) and an occupational therapist may work on maintaining a sitting position (activity) in order to assess consciousness and orientation functions (body function). Therefore, whilst there may be a perceived role overlap with interventions geared to maintaining a sitting position, the clinical reasoning behind each intervention will be different. Using the domains within the ICF would demonstrate the underlying clinical reasoning for different professions working in collaboration on the same activity. 
Expert opinion within healthcare has supported the use of the ICF within stroke rehabilitation. Documentation for assessment and goal setting using the ICF is currently being trialled within the stroke service at the Royal Free Hampstead NHS Trust, UK for use within the MDT. The occupational therapy team have adopted the ICF within the adult services and report they are able to clearly demonstrate and communicate their clinical reasoning and goal setting ${ }^{10}$. In each case, the ICF framework provides headings within the formative assessment. This allows clinicians to demonstrate the many factors impacting on performance including contextual factors, health condition, and activity and participation issues, as identified with the client.

Clinicians also report that students respond to the structure provided by the ICF, as it enables clear demonstration of their clinical reasoning behind intervention and clarifies student learning of the process for intervention.

\section{Limitations}

Whilst the literature provides examples of how the ICF can be used in practice, at present it is essentially descriptive. Therefore there is a need for more robust evidence in order to substantiate claims that the ICF can clarify team roles and demonstrate clinical reasoning within the MDT.

Like health and health-related states, the ICF is itself an evolving process and future work is needed. For example, the core sets are recommended for use in 
pilot studies only at present ${ }^{15}$ and further work is required to determine the efficacy of using these in practice.

A further consideration is that members of the MDT need to learn the new language, framework and classification system of the ICF whilst considering its application to practice. There is also a need to explore change management theory in order to effectively introduce the ICF into clinical practice. Clinicians will need to transpose the ICF language, framework and classification to their existing professional models, definitions and outcome measures, although it is acknowledged that research is currently being undertaken to link the ICF with existing outcome measures and tools ${ }^{19-22}$.

\section{Conclusion}

The evidence from expert opinion and the literature to date, suggests the ICF framework has the potential to clarify MDT roles and demonstrate clinical reasoning within stroke rehabilitation. However, further experiential research is required to evaluate the application in clinical practice, which is being explored by the first author.

\section{Acknowledgements}

The authors would like to thank the Royal Free Hampstead NHS Trust Occupational Therapy Department, Royal Free Hampstead NHS Trust Stroke 
Service, Dr Margaret Mayston and the 2003 MSc Neurophysiotherapy cohort, University College London, UK for their contributions and opinions. Also to $\mathrm{Dr}$ Anita Atwal for her constructive contributions to development of this article.

\section{References}

1. Stroke Unit Trialists' Collaboration. Organised inpatient (stroke unit) care for stroke. The Cochrane Database of Systematic Reviews.2001; Issue 3

2. Langhorne P, Dennis MS. Stroke Units: An evidence-based approach on behalf of the Stroke Unit Trialists' Collaboration. London. BMJ Books. 1998.

3. Intercollegiate Stroke Working Party. National Clinical Guidelines for Stroke ( $2^{\text {nd }}$ ed.) Clinical Effectiveness and Evaluation Unit. London. Royal College of Physicians. 2004.

4. World Health Organisation. International Classification of Functioning, Disability and Health. Geneva. World Health Organisation. 2001.

5. Smith S, Roberts P, Balmer S. Role overlap and Professional Boundaries: Future Implications for Physiotherapy and Occupational Therapy in the NHS. Physiotherapy 2000; 86(8):397 - 400. 
6. Brown GT, Greenwood J Occupational Therapy and Physiotherapy: Similar but separate. British Journal of Occupational Therapy 1999; 62 (4): $163-170$.

7. Stucki G, Cieza A, Ewert T, Kostanjsek N, Chatterji S, Bedirhan Usutn T. Application of the International Classification of Functioning, Disability and Health (ICF) in clinical practice. Disability and Rehabilitation 2002; 24(5): $281-282$

8. Bornman J. The World Health Organisation's terminology and classification: application to severe disability. Disability and Rehabilitation 2004; 26(3): 182 - 188.

9. Stucki G, Bedirhan Ustun T, Melvin J. Applying the ICF for the acute hospital and early post-acute rehabilitation facilities. Disability and Rehabilitation 2005; 27 (7/8): $349-352$

10. College of Occupational Therapists. Guidance for the use of the International classification of functioning, disability and health and the Ottawa charter for health promotion in occupational therapy services. London. College of Occupational Therapists. 2004. 
11. Stucki G, Ewert T, Cieza A. Value and application of the ICF in rehabilitation medicine. Disability and Rehabilitation 2002; 24(17): 932938.

12. Rentsch HP, Bucher P, Domment Nyffeler I, Wolf C, Hefti H, Fluri E, Wenger $\mathrm{U}$, Walti $\mathrm{C}$, Boyer I. The implementation of the International Classification of Functioning, Disability and Health (ICF) in daily practice of Neurorehabilitation: an interdisciplinary project at the Kantonsspital of Lucerne, Switzerland. Disability and Rehabilitation 2003; 25(8): 411 - 421.

13. Steiner W, Ryser L, Huber E, Uebelhart D, Aeschlimann A, Stucki G. Use of the ICF Model as a Clinical Problem-Solving Tool in Physical Therapy and Rehabilitation Medicine. Physical Therapy 2002; 82(11): 1098 -1107.

14. Kearney PM, Pryor J. The International Classification of Functioning, Disability and Health (ICF) and nursing. Journal of Advanced Nursing $2004 ; 46(2) 162-170$

15. Geyh S, Cieza A, Schouten J, Dickson H, Frommelt P, Omar Z, Kostanjsek N, Ring H, Stucki G. ICF Core Sets for Stroke. Journal of Rehabilitation Medicine 2004; Supp 44:135 -141. 
16. Booth J, Hewison A. Role overlap between occupational therapy and physiotherapy during in-patient stroke rehabilitation: an exploratory study. Journal of Interprofessional Care 2002; 16 (1): 31 - 40.

17. Boldt C, Brach M, Grill E, Bethou A, Meister K, Scheuring M, Stucki G. The ICF categories identified in nursing interventions administered to neurological patients with post-acute rehabilitation needs. Disability and Rehabilitation 2005; 27 (7/8): $431-436$

18. Stier-Jarmer M, GrillE, Ewert T, Bartholomeyczik S, Finger M, Mokrusch T, Kostanjsek N, Stucki G. ICF core set for patients with neurological conditions in early post-acute rehabilitation facilities. Disability and Rehabilitation 2005; 27 (7/8): $389-395$

19. Scheuringer M, Grill E, Boldt C, Mittrach R, Mullner P, Stucki G. Systematic review of measures and their concepts used in published studies focusing on rehabilitation in the acute hospital and in early post acute rehabilitation facilities. Disability and Rehabilitation 2005; 27 (7/8): $419-429$

20. Salter K, Jutai JW, Teasell R, Foley NC, Bitensky J. Issues for selection of outcome measures in stroke rehabilitation: ICF Body Functions. Disability and Rehabilitation 2005; 27 (4): $191-207$ 
21. Salter K, Jutai JW, Teasell R, Foley NC, Bitensky J, Bayley M. Issues for selection of outcome measures in stroke rehabilitation: ICF Activity. Disability and Rehabilitation 2005; 27 (6): $315-340$

22. Salter K, Jutai JW, Teasell R, Foley NC, Bitensky J, Bayley M. Issues for selection of outcome measures in stroke rehabilitation: ICF Participation. Disability and Rehabilitation 2005; 27 (9): 507 - 528 